\title{
Many-valued logic: beyond algebraic semantics
}

\author{
Stefano Aguzzoli $\cdot$ Brunella Gerla $\cdot$ Vincenzo Marra
}

(C) Springer-Verlag 2012

The last three decades have witnessed major advances in many-valued logic and related fields. The theory of Łukasiewicz logic and Chang's MV-algebras has flourished, establishing profound connections with other fields of mathematics; Petr Hájek's framework for mathematical fuzzy logic has met with remarkable success, bringing into focus the central rôle of residuation; and the algebraic analysis of substructural logics via residuated lattices has proved itself most fruitful, providing an illuminating bridge between algebra and proof theory. Many-valued logic is here to stay-or so it seems today.

Algebra was a key ingredient of these developments: indeed, many-valued logic has been traditionally investigated through its algebraic semantics. And yet, logic is not just algebra. A distinctive feature of logic is its concern with the relationship between syntax (language) and semantics (the world). From this point of view, algebra belongs to the syntactic aspects of the subject. One may go as far as saying that the locution "algebraic semantics", though established, is in fact oxymoronic. While Heyting algebras are the equivalent algebraic semantics of the intuitionistic propositional calculus, a genuinely semantic

\footnotetext{
S. Aguzzoli

Dipartimento di Scienze dell'Informazione, Università degli Studi di Milano, Milan, Italy

e-mail: aguzzoli@dsi.unimi.it

B. Gerla $(\bowtie)$

Dipartimento di Informatica e Comunicazione, Università degli Studi dell'Insubria, Varese, Italy

e-mail: brunella.gerla@uninsubria.it

\section{Marra}

Dipartimento di Matematica Federigo Enriques, Università degli Studi di Milano, Milan, Italy

e-mail: vincenzo.marra@unimi.it
}

understanding of intuitionism must rely on relational, Kripke-style structures. When compared on these grounds, many-valued logic appears to lag behind inuitionistic and modal logics. This special issue of Soft Computing is intended as a contribution to the major research project of developing a deeper understanding of the meaning of many-valued logical systems, reaching beyond algebraic semantics. The collection of essays we present here is a follow-up to the third instalment of the biennial conference series ManyVal, held in Varese, Italy, during May 2010. The meeting gathered about 40 researchers with varied backgrounds, but all sharing a keen interest in the semantics of many-valued logics. Although the papers in this special issue range from the foundational to the technical, it will transpire that at some level they are all related to the theme of the conference.

Two papers in the present collection address issues of broad significance explicitly. Didier Dubois's contribution discusses an embedding of Kleene and Belnap many-valued logics - sporting three and four truth values, respectively-into a system of epistemic logic. This is in line with the origins of these many-valued systems, which were conceived by their inventors as logics of knowledge and contradiction. Beyond the specific results it illustrates, the paper may be construed as a thought-provoking essay within the major debate on the relationship between vagueness (many-valued logic) and ignorance (epistemic logic). By contrast, the paper by Thomas Vetterlein focusses on theories of vagueness in themselves. Using as testing grounds such classical topics as the sorites paradox and the syllogism, the author advances and defends his own view that a central challenge in connection with reasoning under vagueness is to find models for the varying levels of granularity relevant to a particular context. He argues that this leads to rejecting the view that any single formal 
system may be the appropriate one to reason in the presence of vagueness.

Other contributions in this issue offer more specialised results. Three papers are concerned with MV-algebras, the equivalent algebraic semantics of Łukasiewicz infinitevalued propositional logic. In accordance with the spirit of this issue, each one of these three papers probes research directions which may eventually lead from pure algebraic semantics to a broader understanding of the meaning of Łukasiewicz's time-honoured many-valued system. Ioana Leuştean proves an MV-algebraic version of the classical Hahn-Banch extension theorem. To this end she introduces seminorms on MV-algebras, generalising states and orderunit norms of lattice-ordered Abelian groups with a strong order unit. Seminorms on MV-algebras are related to MV-algebraic generalisations of imprecise probabilities in the sense of Walley, as the author points out. Tomáš Kroupa's paper breaks new ground by advancing a definition of belief function for free finitely generated MV-algebras on the basis of an in-depth analysis of the Boolean notion introduced by Dempster and Shafer in the 1970s. The MValgebras in question are the Lindenbaum algebras of Łukasiewic logic over finitely many propositional variables; hence the author's treatment amounts to one of the first attempts at extending Shafer's theory of evidence to manyvalued events-events described by formulae of a manyvalued logic. Readers will appreciate the mathematical richness of this nascent theory, whose tools range from topological hyperspaces to the Choquet integral-not to mention, of course, the representation theory for MV-algebras. The paper by Tommaso Flaminio, Lluís Godo, and Enrico Marchioni is tightly related to Kroupas' contribution with its quite different mathematical flavour notwithstanding. Indeed, the authors investigate generalisations of possibility and necessity measures to MV-algebras of functions (MV-clans). The connection with Kroupas' work becomes evident once it be recalled that, in the Boolean setting, necessity measures are a special case of belief functions. At a general level, an important feature of the authors' contribution is to bring to the fore the intimate relationship between the non-additive notions of possibility and necessity measures, and the tropical geometry based on the idempotent semirings max-plus and min-plus of real numbers.
With the remaining two papers of this issue, we turn to algebraic structures other than MV-algebras related to many-valued reasoning. The paper by George Metcalfe and Christoph Röthlisberger deals with deduction rules admissible by a given logic, i.e. rules that produce no new theorems when added to the logic. While in classical logic each admissible rule is derivable, and hence carries no new information, in non-classical systems it is common to have admissible, non-derivable rules. Their understanding can provide important insights into the nature of the logic at hand. The authors offer a characterisation of the quasiidentities admissible by De Morgan algebras, that is, bounded distributive lattices endowed with an involutive negation that satisfy De Morgan's Laws. These structures are the algebraic counterparts of the four-valued logic of Belnap that is featured in Dubois' paper. For algebraizable logics, quasi-identities are the algebraic correspondent of admissible rules; it is known, however, that De Morgan algebras do not algebraize any logic. Hence the authors' treatment is algebraic. And so is the one given by Maria Emilia Della Stella and Cosimo Guido in their own paper: here, the starting point is Helena Rasiowa's algebraic approach to non-classical logics, based on implicative algebras. The underlying notion driving the authors' development, however, is the adjunction between implication and conjunction (residuation) that is a fundamental feature of both classical and intuitionistic logic, and also lies at the heart of the theory of residuated lattices. Under the assumption of lattice-theoretic completeness, residua and dual residua always exist, hence the authors focus on this case. Given this setting, the core of the paper consists of an investigation of the algebraic counterparts of the implicator functions considered in the literature on fuzzy logic, in the broad sense of the term.

As Guest Editors, we are proud to present to the reader this collection of interesting contributions to the general problem of understanding the deeper meaning of manyvalued logical systems. This special issue would not have seen the light without the efforts of the authors and of the anonymous referees, to whom our gratitude goes.

Milan, 10.1.2012

The Guest Editors 